\title{
SCALE/MAVRIC calculation of dose rates measured for a gamma radiation source in a thick-walled transport and storage cask of ductile cast iron with lead inserts
}

\author{
Werner Baumgarten ${ }^{1, a}$, Holger Thiele ${ }^{1}$, Benjamin Ruprecht ${ }^{1}$, Peter-W. Phlippen ${ }^{2}$, Luc Schlömer ${ }^{2}$ \\ ${ }^{1}$ Bundesamt für kerntechnische Entsorgungssicherheit (BfE), Postfach 100149, 38201 Salzgitter, Germany \\ ${ }^{2}$ WTI Wissenschaftlich-Technische Ingenieurberatung GmbH, Karl-Heinz-Beckurts-Straße 8, 52428 Jülich, Germany
}

\begin{abstract}
Dose rate calculations are important for judging the shielding performance of transport casks for radioactive material. Therefore it is important to have reliable calculation tools. We report on measured and calculated dose rates near a thick-walled transport and storage cask of ductile cast iron with lead inserts and a Co-60 source inside. In a series of experiments the thickness of the inserts was varied, and measured dose rates near the cask were compared with SCALE/MAVRIC 6.1.3 and SCALE/MAVRIC 6.2 calculation results. Deviations from the measurements were found to be higher for increased lead thicknesses. Furthermore, it is shown how the shielding material density, air scattering and accounting for the floor influence the quality of the calculation.
\end{abstract}

\section{Introduction}

The calculation of dose rates behind multiple shielding layers of material is highly relevant for radiation shielding assessment tasks within package design approval procedures. Thus, it is important that the underlying calculation methods work as accurate as possible and that they are validated against experimental data. A series of experiments was performed and evaluated by WTI (Wissenschaftlich-Technische Ingenieurberatung $\mathrm{GmbH}$ ) allowing to check the accuracy of such calculation tools (cp. [3]). The paper reports on modelling and dose rate calculations with SCALE/MAVRIC 6.1.3 [1] and SCALE/MAVRIC 6.2 [4] by BfE and how the results compare to the measured data.

\section{Experimental Setup}

The experimental setup consisted of a freely moveable gamma radiation detector and a cylindrical transport and storage cask of ductile cast iron standing on a concrete floor (Fig. 1). Through small holes in the lid of the closed cask a Co-60 gamma source was placed inside. The shielding properties of the cask were changed by inserting hollow lead cylinders with different thicknesses between $20 \mathrm{~mm}$ and $80 \mathrm{~mm}$.

Gamma energy dose rates (kerma-free-in-air) were measured using different ionization chambers connected to a universal dosimeter of type PTW UNIDOS. By varying the position of the source as well as the thickness of the inserts different configurations were considered.
The gamma energy dose rates were measured at radial distances of $16.5 \mathrm{~cm}, 50 \mathrm{~cm}, 100 \mathrm{~cm}, 150 \mathrm{~cm}$, and $200 \mathrm{~cm}$ from the surface of the cask body in planes at the top, the middle and the bottom. Due to the high source activity of about $1 \mathrm{TBq}$, dose rates could be measured accurately even for the highest lead thickness.

In order to ensure homogeneous shielding properties of the cast iron wall and the lead between source and detector these structures were checked for material errors, e.g. cavities, by gamma radiography before the experiments were carried out. Additionally, the real cast iron and lead density were measured after the measurement series had been conducted.

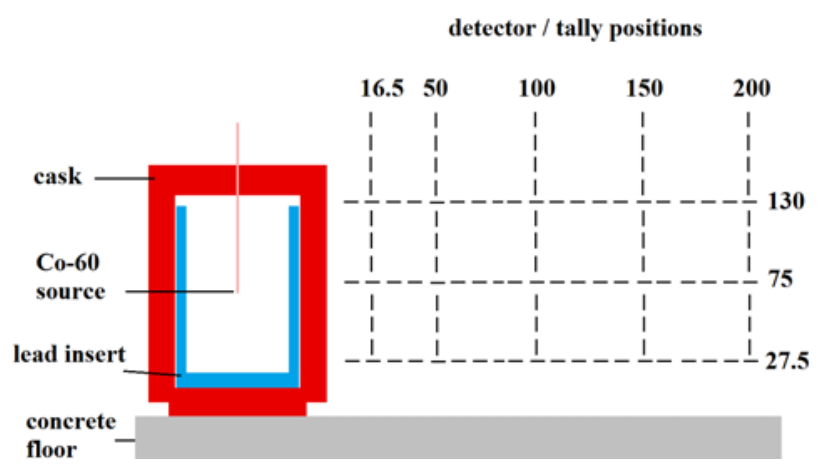

Fig. 1: Cross-section view of the storage cask and the detector positions (dimensions in $\mathrm{cm}$ ).

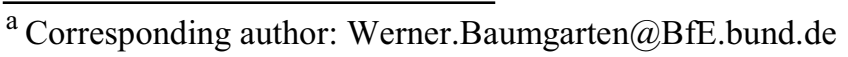




\section{Calculation Methods and Model}

Co-60 predominantly emits gamma photons with energies of $1.17 \mathrm{MeV}$ and $1.33 \mathrm{MeV}$, which can only be modelled directly in programs working with continuous energies. Thus, in order to model the Co-60 source in multi-group calculations an energy conserving gamma spectrum was calculated for the 47 gamma group library available in the SCALE 6 package with ORIGEN-ARP 6.1. Dose rates were then calculated with the MAVRIC [2] sequence of SCALE 6.1.3 using point tallies and the v7$200 \mathrm{n} 47 \mathrm{~g}$ shielding library based on ENDF/B-VII data. However, SCALE/MAVRIC 6.2 offers the possibility to use continuous energy cross sections in the transport calculation which allowed us to model the Co-60 source without any pre-processing of the spectrum. In our model the source definition relies on the implemented singlenuclide spectrum.

A cross section of the cask model and the different detector positions are shown in Fig. 1. The grid geometry for the calculation in MAVRIC that covers the cask consisted of 60 bins for each of the $\mathrm{x}$ - and $\mathrm{y}$-direction, and 100 bins for the z-direction (to the top), while the concrete floor was modelled with 10 bins in $\mathrm{x}, \mathrm{y}$ and $\mathrm{z}$ direction. The source itself was modelled by a small cylinder of $0.4 \mathrm{~cm}$ in diameter and height. Monte-Carlo calculations with SCALE/MONACO code were conducted with 1600 batches of 1000 particles resulting in typical relative standard deviations of up to $0.7 \%$ of the calculated dose rates.

The dose rates gained from the model are influenced by several parameters. In this paper we focus on the lead thickness, the floor, the density of the shielding cask material, air in the free volume and the type of the energy library used to model the Co-60 source and the photon transport. For these model simulations, if not stated otherwise, the following setup has been used. The source was placed in the centre of the cask at $75 \mathrm{~cm}$ height and the tallies (virtual detector positions) were placed in the middle plane also at $75 \mathrm{~cm}$ height (Fig. 1). Energy dose rates were calculated using the appropriate ICRU-57 flux to dose rate conversion factors integrated in SCALE. The uncertainties of the Co-60 source activity (2.4\%) and the calibration of the dosimeter $(2.0 \%)$ result in a systematic error of $4.4 \%$. The relative error of $1.3 \%$ is composed of the standard deviation of the repeated measurements $(0.5 \%)$, the exact positioning of the source $(1.0 \%)$, and the Monte-Carlo simulation results $(0.7 \%)$.

\section{Results}

The relative deviation of the calculated dose rates from the measured dose rates changes only negligibly when the detector distance from the cask is varied. This is shown in Fig. 2 for the results obtained using SCALE/MAVRIC 6.1.3 with the multi-group shielding library. However, the deviation from the measured dose rate significantly increases when thicker lead insets are used, which can also be seen in Fig. 3 where the distance-averaged deviations are plotted vs. the lead thickness. The multi-group model underestimates the dose rate by up to $25 \%$ at $80 \mathrm{~mm}$ lead thickness (Fig. 2) whereas the underestimation is as low as about $7 \%$ when no lead is present. For the SCALE/MAVRIC 6.2 model with continuous energy library a similar behaviour is observed, see Fig. 3. Yet, an underestimation of the dose rate is only observed for lead thicknesses above $40 \mathrm{~mm}$. Without lead and for the $20 \mathrm{~mm}$ inset the dose rate is even overestimated.

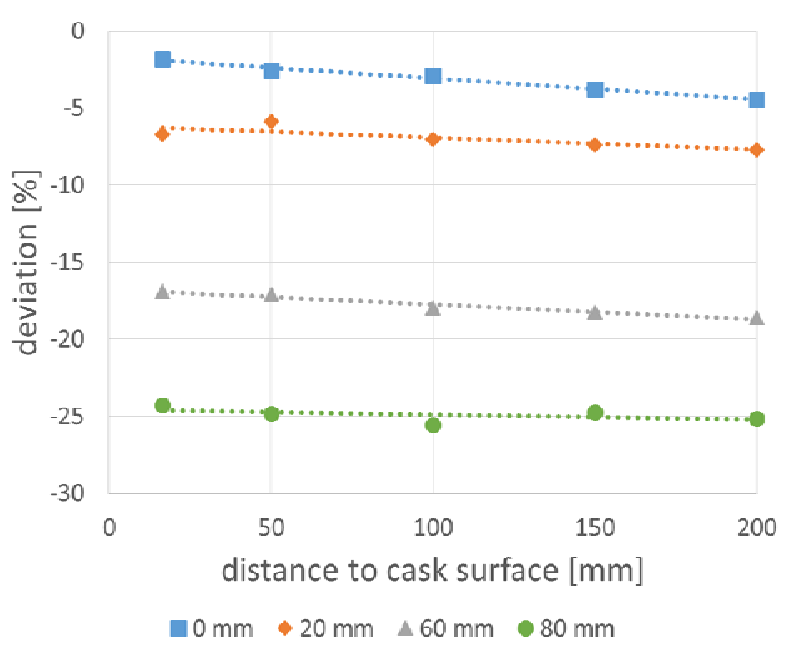

Fig. 2: Deviations from the measured dose rates in SCALE/MAVRIC 6.1.3 multi-energy group calculations with different lead thicknesses $(0 \mathrm{~mm}, 20 \mathrm{~mm}, 60 \mathrm{~mm}$ and $80 \mathrm{~mm}$ ) and detector positions.

Nevertheless, the slopes of the trending lines in Fig. 3 are comparable. Additional tests have clearly shown that SCALE 6.2 with multi-group energy model settings leads to exactly the same results as SCALE 6.1.3 (not shown here). All in all, the clear trend for underestimation of the gamma dose rates when thick-walled shields are employed has to be considered in future studies with both SCALE 6.1.3 and SCALE 6.2 calculations.

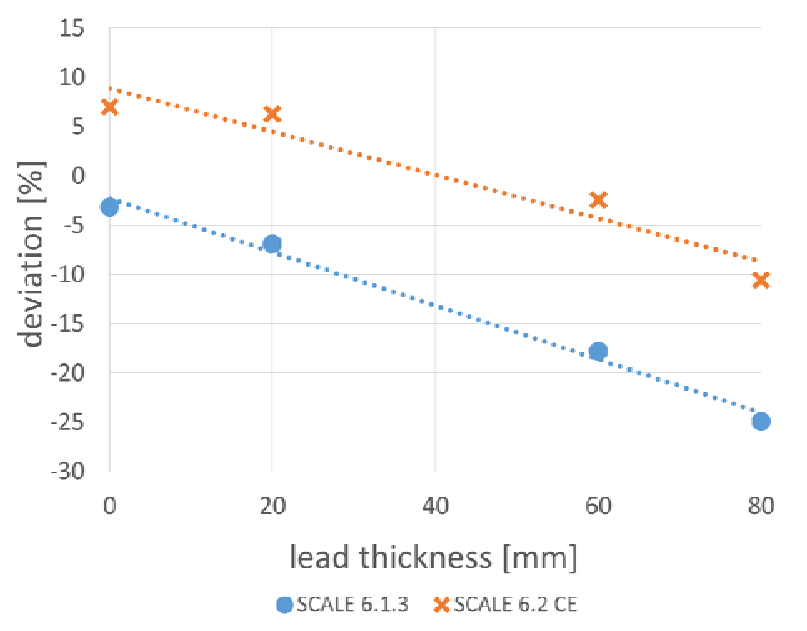

Fig. 3: Average deviation from measured dose rates by a multi-group energy model (SCALE 6.1.3, blue line) and a continuous energy model (SCALE 6.2, orange line).

The uncertainty in the exact knowledge of the density of the shielding material influences the results of the 
simulation. To quantify this influence, the density of the shielding material of the cask was changed from $7.07 \mathrm{~g} / \mathrm{cm}^{3}$ to $7.00 \mathrm{~g} / \mathrm{cm}^{3}$, i.e. was reduced by $1 \%$. Fig. 4 shows that on average this $1 \%$ decrease of the density results in an increase of the dose rate of about $5.0 \%$, regardless of the thickness of the lead insert. Thus, the exact knowledge of the density of the shielding material is crucial to gain correct dose rate results in the model.

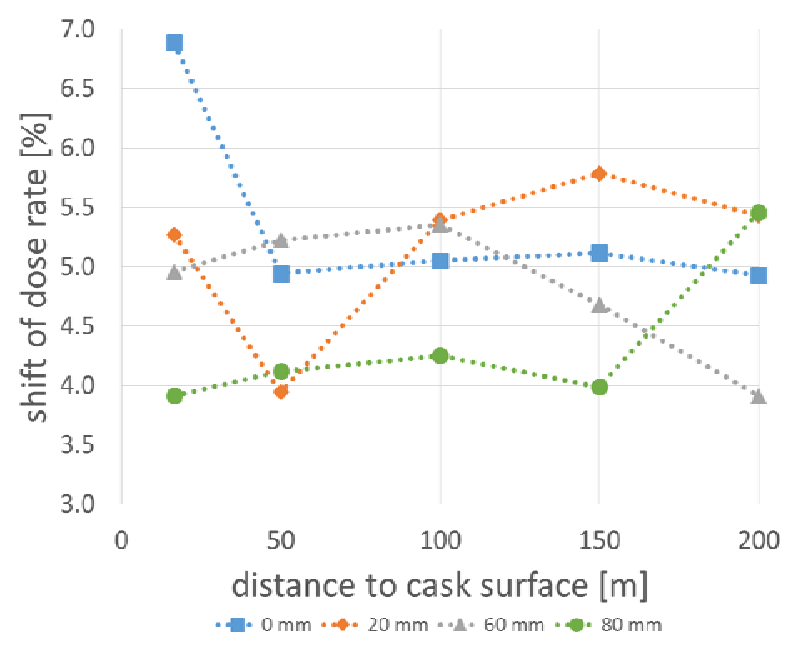

Fig. 4: Influence of changing the shielding material density from $7.07 \mathrm{~g} / \mathrm{cm}^{3}$ to $7.00 \mathrm{~g} / \mathrm{cm}^{3}$ on the calculation results for different lead thicknesses $(0 \mathrm{~mm}, 20 \mathrm{~mm}, 60$ $\mathrm{mm}$ and $80 \mathrm{~mm}$ ). Calculated with SCALE 6.1.3.

Air, as a weak absorber and scatterer of gamma radiation, may also affect the resulting dose rate. However, our calculations show that at short distances of not more than $200 \mathrm{~cm}$, air has only little influence of about $1 \%$ on the results, see Fig. 5, which is not significant within two standard deviations of the Monte-Carlo calculations.

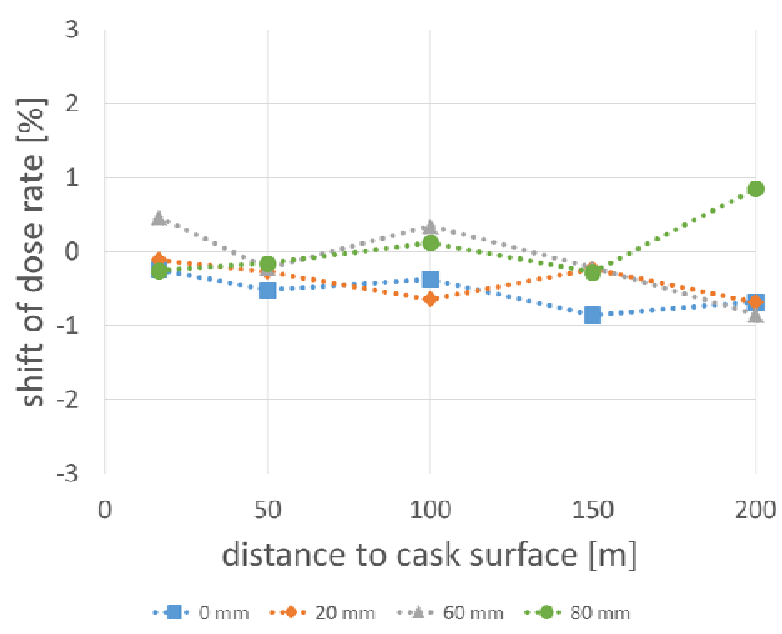

Fig. 5: The effect of including air into the models at various lead insert thicknesses $(0 \mathrm{~mm}, 20 \mathrm{~mm}, 60 \mathrm{~mm}$ and $80 \mathrm{~mm}$ ) is between $-1 \%$ and $1 \%$. Calculations performed with SCALE 6.1.3.

We also studied to which extend the concrete floor affects the dose rate results in the model. Whereas the floor was included in all previous models, we ran the same simulations as before but without the floor, and compared the dose rates. For the detector positions in the middle plane the results show that accounting for the floor increases the dose rates by up to $4 \%$ (see Fig. 6). In an additional set of models, we also included the detector positions right above the floor on the lower plane in Fig. 1.

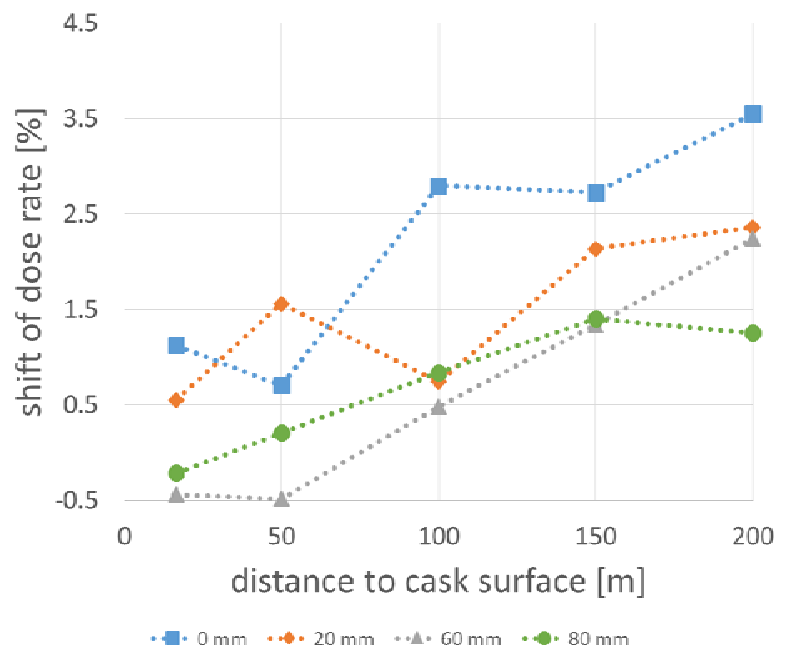

Fig. 6: Increase of dose rates in the middle plane (Fig. 1) when accounting for the concrete floor, under various lead insert thicknesses $(0 \mathrm{~mm}, 20 \mathrm{~mm}, 60 \mathrm{~mm}$ and 80 $\mathrm{mm})$. Calculations performed with SCALE 6.1.3.

At these positions the dose rate turns out to be much more sensitive to the absence of the floor. From Fig. 7 it can be seen that the presence of the floor contributes up to about $6 \%$ of the value of the dose rates, as measured by the virtual detectors.

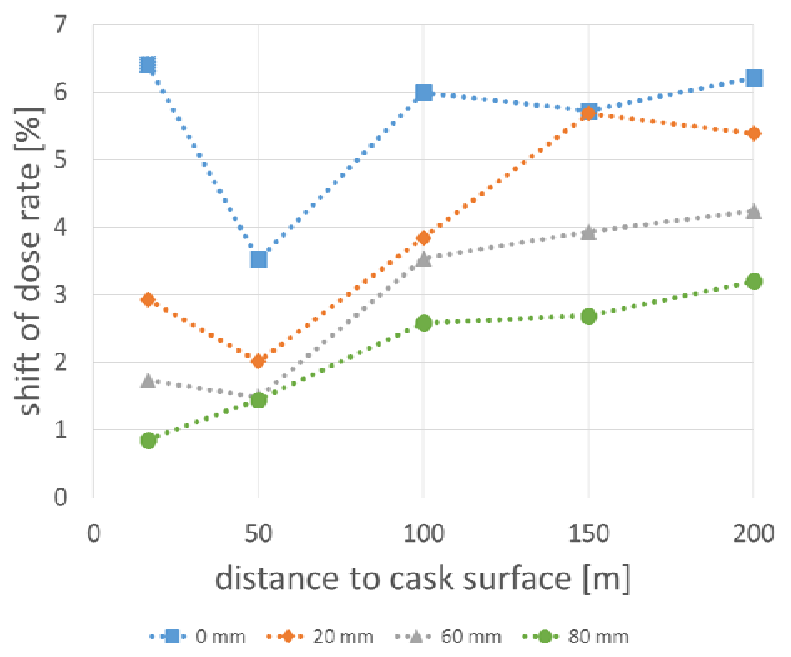

Fig. 7: Increase of dose rates in the lower plane (Fig. 1) when accounting for the concrete floor, studied for various lead insert thicknesses $(0 \mathrm{~mm}, 20 \mathrm{~mm}, 60 \mathrm{~mm}$ and $80 \mathrm{~mm}$ ). Calculations performed with SCALE 6.1.3.

The large influence of the floor on the calculated dose rates can be explained by considering that there is a 
scattering of the gamma radiation that interacts with the concrete.

\section{Discussion and Conclusion}

The goal of the study was to determine the accuracy of dose rate calculations for thick-walled transport and storage casks using two versions of SCALE/MAVRIC. Precise modelling of the different configurations and dose rate comparisons were possible due to an elaborate experimental setup and well-documented measurement series. Overall, the results of our SCALE 6.1.3 model fit well the results described in an earlier study of the same cask, where analyses identify the variability of the group wise modelling of the Co-60 source as one significant parameter causing the observed deviations to the measurements and the MCNP-results presented in [3]. The results of the present work do not fully support this finding. Our analysis has shown that continuous energy calculations with the release version of SCALE 6.2 does not completely resolve the problem of the observed underestimations. For a Co-60 source switching from a SCALE/MAVRIC full multi-group model to a continuous energy model shifts the dose rate deviations by about $10 \%$ to the positive direction, but does not significantly mitigate the trend for underestimation. It is worth noting that this trend is less pronounced in calculations conducted with the continuous energy code MCNP [3]. Furthermore, additional SCALE 6.2 models with continuous energy cross sections used in the transport calculation but a multi-group source definition yield dose rates between those resulting in the deviations shown in Fig. 3. Hence, both, the change from the multi-group (ENDF/B-VII.0) to the continuous energy cross section library (ENDF/B-VII.1) and from the multi-group to the continuous energy source definition contribute to these differences with similar magnitude.

These effects must be accounted for in shielding assessments with SCALE/MAVRIC in multi-group and continuous mode for package design approval procedures. Careful code validation for the application case is still essential when using SCALE/MAVRIC for dose rate calculations in geometries with thick-walled gamma radiation shields and an appropriate quantitative correction of the results may be necessary. Apart from that, the present paper has shown that parameters such as accounting for the floor, which scatters the gamma radiation and the density of the cask shielding material crucially affect the deviation of the measured dose rates, up to $6 \%$ for the floor and $5 \%$ for a hypothetical change of $1 \%$ in the density of the cast iron. At the short distances used in this measurement setup, air does not play an important role for the dose rates observed.

\section{References}

[1] SCALE: A Comprehensive Modeling and Simulation Suite for Nuclear Safety Analysis and Design, ORNL/TM-2005/39, Version 6.1, Oak Ridge National Laboratory, June 2011
[2] D. E. Peplow, Monte Carlo Shielding Analysis Capabilities with MAVRIC, Nucl. Technol. 174(2), 289313, May 2011.

[3] L. Schlömer, S. Tittelbach, P.-W. Phlippen, J. Pillath, W. Filbert, Validation and Benchmarking of Calculation Methods for Photon and Neutron Transport at Cask Configurations, PHYSOR 2014, 28. September -

3. October 2014, Kyoto, Japan

[4] SCALE Code System ORNL/TM-2005/39, Version 6.2, Oak Ridge National Laboratory, April 2016 